\title{
Stability of a power law relation between characteristics of earthquakes and electric precursors
}

\author{
E. Dologlou \\ Solid State Section, Department of Physics, University of Athens, Panepistimiopolis, Zografos 15784 Athens, Greece \\ Correspondence to: E. Dologlou (edologl@phys.uoa.gr)
}

Received: 21 February 2012 - Revised: 17 March 2012 - Accepted: 19 March 2012 - Published: 29 May 2012

\begin{abstract}
New data were used to test the credibility of a previously reported power law relation between the stress drop of earthquakes and the lead time of precursory SES. Here, we found that the critical exponent of this power law is very sensitive and remains stable around 0.33 only for appropriate sets of data. This value is in full agreement with the reported one in literature for critical phenomena. That means this power law is not an artifact, but probably implies that real physical dynamic processes evolving to criticality are present in the pre-focal area when the SES is emitted. An attempt to advance the underlying physics of the interconnection of the stress drop and the lead time of the precursory SES is still in progress.
\end{abstract}

\section{Introduction}

The new approach that large earthquakes can be understood as a critical phase transition in the frame of the statistical physics finds support in recent observations that rupture in heterogeneous media is a critical phenomenon. The dimension of the area where the preparation processes develop and premonitory patterns can be observed greatly exceeds source size of main shock (see the summary in Keilis-Borok, 2003, Table 1.2). As the area approaches the critical point, long-range correlations, which are a general feature of complex systems in a near-critical state, emerge and nonlinear procedures following power laws govern the evolving earthquake dynamic processes (Telesca et al., 2005; Teotia and Kumar, 2011) and consequently its associated precursors. The existence of power law is a necessary condition, but not enough to characterize a critical state. For instance, both artificial noises and precursory SES activities (which are low frequency, $<1 \mathrm{~Hz}$, transient changes of the Earth's electrotelluric field), follow power law behaviour of complex systems, but only the SES activities exhibit critical dynamics (Varotsos et al., 2002, 2011a).

Seismic Electric Signals (SES) have been detected prior to large earthquakes in Greece for the last 3 decades (Varotsos and Alexopoulos, 1984a, b; Varotsos et al., 1986, 1988, 1993a, b). A possible SES generation mechanism is the following: rocks in the Earth's crust contain various solids with intrinsic (Varotsos and Alexopoulos, 1978, 1979, 1984c) and extrinsic lattice defects, which appear for charge compensation, particularly in ionic solids (Varotsos and Alexopoulos, 1981) when doped with aliovalent impurities. Electric dipoles that are formed between a portion of these defects and nearby impurities can change orientation in a cooperative way when increasing applied stress reaches a critical value $P_{\mathrm{cr}}$, thus, giving rise to a transient signal, provided that upon increasing stress, the relaxation time of dipoles decreases, which means negative migration volume (Varotsos and Alexopoulos, 1980, 1986). This signal constitutes an SES and it is characterized by critical dynamics and a series of such signals is termed SES activity (Varotsos and Alexopoulos, 1986; Varotsos et al., 2011a).

The SES amplitude $E=\Delta V / l$ (with $\Delta V$ the potential difference measured between to points on the ground at a distance $l$ ) and the magnitude $M$ of the impending earthquake are connected through the experimental relation:

$\log E=\mathrm{a} M+b$

where $\mathrm{a} \approx 0.3-0.4$ and $b$ is a constant, depending on the geoelectrical structure around the measuring site. Usually, a-value is around 0.33 (Varotsos and Alexopoulos, 1984a, p. 91). The SES emission signals the entrance of the prefocal area into a critical stage. Thus, the power law relation expressed by Eq. (1) is reminiscent of the theory of critical phenomena (Varotsos and Alexopoulos, 1984a, b). 
Table 1. The USGS reported date and magnitude of the 3 main shocks along with their seismic moment, range of values in the dimensions of their aftershock area $L$ and $W$, corresponding calculated values for $\Delta \sigma_{B}$, the critical exponent $\alpha$ and the lead time $\Delta t$. Mean values are also given for each event.

\begin{tabular}{|c|c|c|c|c|c|c|c|c|}
\hline$n_{0}$ & $\begin{array}{l}\text { Date } \\
\text { yy/mm/dd }\end{array}$ & $M$ & $\begin{array}{r}M_{O} \\
\left(10^{23} \text { dyn } \mathrm{cm}\right)\end{array}$ & $\begin{array}{r}L \\
(\mathrm{~km})\end{array}$ & $\begin{array}{r}W \\
(\mathrm{~km})\end{array}$ & $\begin{array}{r}\Delta \sigma_{B} \\
\text { (bars) }\end{array}$ & $\alpha$ & $\begin{array}{r}\Delta t \\
\text { days }\end{array}$ \\
\hline 1 & $\begin{array}{l}11 / 07 / 19 \\
\text { Mean value }\end{array}$ & $5.1(\mathrm{mb})$ & 8.1 & $\begin{array}{r}19-21 \\
20\end{array}$ & $\begin{array}{r}4-5 \\
4.5\end{array}$ & $\begin{array}{r}2.99-1.85 \\
2.42\end{array}$ & $\begin{array}{l}0.321-0.334 \\
0.327 \pm 0.01\end{array}$ & 54 \\
\hline 2 & $\begin{array}{l}\text { 11/08/07 } \\
\text { Mean value }\end{array}$ & $5.0\left(M_{\mathrm{W}}\right)$ & 3.5 & $\begin{array}{r}21-23 \\
20\end{array}$ & $\begin{array}{r}9-11 \\
10\end{array}$ & $\begin{array}{r}0.33-0.20 \\
0.265\end{array}$ & $\begin{array}{l}0.406-0.395 \\
0.399 \pm 0.01\end{array}$ & 73 \\
\hline 3 & $\begin{array}{l}11 / 09 / 14 \\
\text { Mean value }\end{array}$ & $5.0(\mathrm{mb})$ & 5.0 & $\begin{array}{r}15-17 \\
16\end{array}$ & $\begin{array}{r}10-12 \\
11\end{array}$ & $\begin{array}{r}0.42-0.66 \\
0.52\end{array}$ & $\begin{array}{l}0.387-0.369 \\
0.378 \pm 0.01\end{array}$ & 111 \\
\hline
\end{tabular}

In a recent paper (Dologlou, 2011) the SES generation mechanism was found to be explained on the basis of a thermodynamical model, known as the $c B \Omega$ model (where $B$ is the isothermal bulk modulus, $\Omega$ the mean atomic volume per atom and $c^{\text {act }}$ is a dimensionless constant), which interconnects defect parameters and bulk properties in solids (Varotsos, 1976, 2007).

An alternative model for the SES emission is the electrokinetic effect (Ishido and Mizutani, 1981). In this case, if the phenomenon is considered in terms of criticality, the appropriate exponent of the power law relation is 0.31 (Surkov et al., 2002), being in excellent agreement with the value $(\mathrm{a} \sim 0.33)$ reported earlier on the basis of empirical data by Varotsos and Alexopoulos (1984a).

The interconnection between SES and impending earthquake characteristics has been investigated in a series of papers. Precisely, a robust power law relation with a critical exponent of 0.33 has been found to interrelate the lead time $\Delta t$ (which is the time difference between the SES emission and the associated earthquake occurrence) and the stress drop (which is the difference between the stress state at a point on a fault before and after the occurrence of the earthquake) of the forthcoming earthquake (Dologlou, 2009, 2010).

Here, we investigate the obedience of new data to this power law relation as well as the sensitivity of the critical exponent to any additional possible pairs of SES and earthquakes.

\section{Data used}

The aforementioned interconnection of SES lead time $\Delta t$ and earthquake stress drop $\Delta \sigma$ through a power law relation with an exponent value of 0.33 has been found by Dologlou $(2009,2010)$ using data from 1981 to 2010 from precursory SES signals and large earthquakes (see Table 1, Dologlou, 2010), for which Brune's stress drop (Brune, 1970, 1971) values were either reported or derived through a special technique based on the dimensions of the aftershock area and seismic moment (Kiratzi et al., 1991). Here, we deal with the largest earthquakes that occurred in Greece within 5 months after the detection of new SES activity, which was recorded at PIR station (blue triangle in Fig. 1) on 2526 May 2011 (Skordas et al., 2012). We note that the maximum time lag between a SES activity and impending earthquake does not exceed 5 months (Varotsos et al., 2011b). Three events with $M \geq 5$ are reported by USGS in the area $(36-41)^{\circ} \mathrm{N},(19-25)^{\circ} \mathrm{E}$ for the period 26 May 2011 to 26 November 2011: the first one on 19 July 2011 with $\mathrm{mb}=$ 5.1 and epicentre $37.21^{\circ} \mathrm{N} 19.92^{\circ} \mathrm{E}$; the second on $7 \mathrm{Au}-$ gust 2011 with $M_{\mathrm{W}}=5.0$ and epicentre $38.44^{\circ} \mathrm{N} 21.83^{\circ} \mathrm{E}$; and the third on 14 September 2011 with $\mathrm{mb}=5.0$ and epicentre $37.19^{\circ} \mathrm{N} 22.05^{\circ}$ E. (see Table 1 and Figs. 1 and 2). We also checked that no other SES activity was reported during the 5 month period prior to the first earthquake on 19 July 2011. Thus, one of the 3 above mentioned events must be associated with this SES activity.

The Brune's stress drop values for these earthquakes were calculated through the formula of Hanks and Wyss (1972):

$\Delta \sigma_{B}=0.44 M_{o} / r^{3}$

where $M_{o}$ is the seismic moment and $r$ the radius for a circular fault. The estimation of the radius $r$ was obtained by the application of the aftershock area technique (Kiratzi et al., 1991), which is described in detail by Dologlou (2009). For the determination of the aftershock areas, we used the earthquakes reported by the National Observatory of Athens (NOA), Greece (http://www.gein.noa.gr/services/Noa_cat/CAT2003.TXT).

Precisely, we considered all events with $M_{\mathrm{L}} \geq 3$ that occurred in a 3 month period after each main shock (Figs. 1 and 2 red stars), respectively and in the following areas: $[19-21)^{\circ} \mathrm{N},[36.5-38.5)^{\circ} \mathrm{E}$ for the first main shock (Fig. 1, solid green circles); $[21-23)^{\circ} \mathrm{N},[38-39)^{\circ} \mathrm{E}$ for the second (Fig. 1, open circles); and $[21-22.5)^{\circ} \mathrm{N},[36.5-38)^{\circ} \mathrm{E}$ for the third one (Fig. 1, open squares). The aftershock area $S=L \times W$, where $L$ is the length and $W$ the width in $\mathrm{km}$ (Table 1), is recognized as a cluster of events around each main shock (Fig. 1). Ellipses, one solid (no. 1) and two 


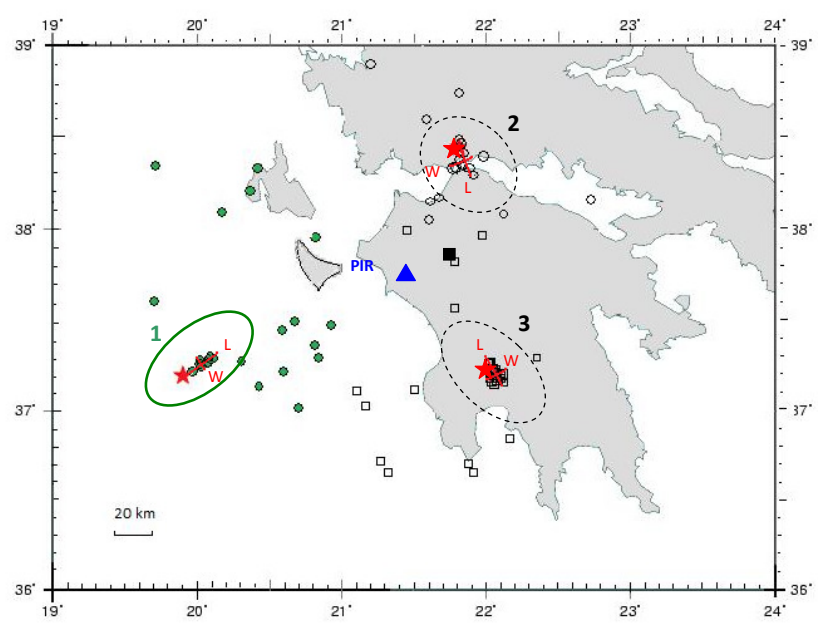

Fig. 1. Map of Greece, with the distribution (forming a cluster) of aftershocks with $M_{\mathrm{L}} \geq 3$ reported by NOA for a three month period after each main shock listed in Table 1. Ellipses, one solid (no. 1) and two dashed (no. 2 and 3), show the location of each cluster with dimensions $\mathrm{W}$ and $L$ for the corresponding aftershock area, while red stars numbered as 1, 2 and 3 denote the epicentres of the associated main shocks of Table 1 . The epicenters of aftershocks are denoted by solid green circles for no. 1 event, open circles for no. 2 event and open squares for no. 3 event. A blue triangle depicts the location of the SES station PIR.

dashed (no. 2 and 3), are used to show on the map of Greece (Fig. 1) the location of each cluster corresponding to the main shocks 1, 2 and 3, respectively as numbered in Table 1. The radius $r$ is calculated from the equation $S=\pi r^{2}$.

The seismic moments $M_{o}$ for the three main shocks are estimated through the formulas: $\log \left(M_{o}\right)=1.73 \mathrm{mb}+15.09$ (Chen et al., 2007) or $\log \left(M_{o}\right)=1.5\left(M_{\mathrm{W}}+10.7\right)$ (Hanks and Kanamori, 1979). We note that for the main shocks no. 2 and no. 3, the reported magnitude by National Observatory of Athens is $M_{\mathrm{L}}=4.8$ and $M_{\mathrm{L}}=4.5$, respectively, which leads to seismic moment values of $M_{o}=2 \times 10^{23} \mathrm{dyn} \mathrm{cm}$ and $M_{o}=0.87 \times 10^{23} \mathrm{dyn} \mathrm{cm}$. For the calculation of stress drop $\Delta \sigma_{B}$ we used Eq. (2).

Since in this study we deal with one new SES activity and three possible corresponding main events, three pairs of lead time $\Delta t$ and stress drop $\Delta \sigma_{B}$ were investigated. We separately inserted each pair into the already obtained power law relation $\Delta \sigma_{B}=8.17 \Delta t^{-0.328}$ based on previous data (Table 1, Dologlou, 2010) and the resulting new exponents $\alpha$ along with their errors are given in Table 1 . The seismic moment $M_{o}$, the stress drop $\Delta \sigma_{B}$, the aftershock dimensions $L$ and $W$ and their error range and mean values, the date magnitude and lead time $\Delta t$ for each main event, numbered as no. 1, 2 and 3 are also presented in Table 1 .

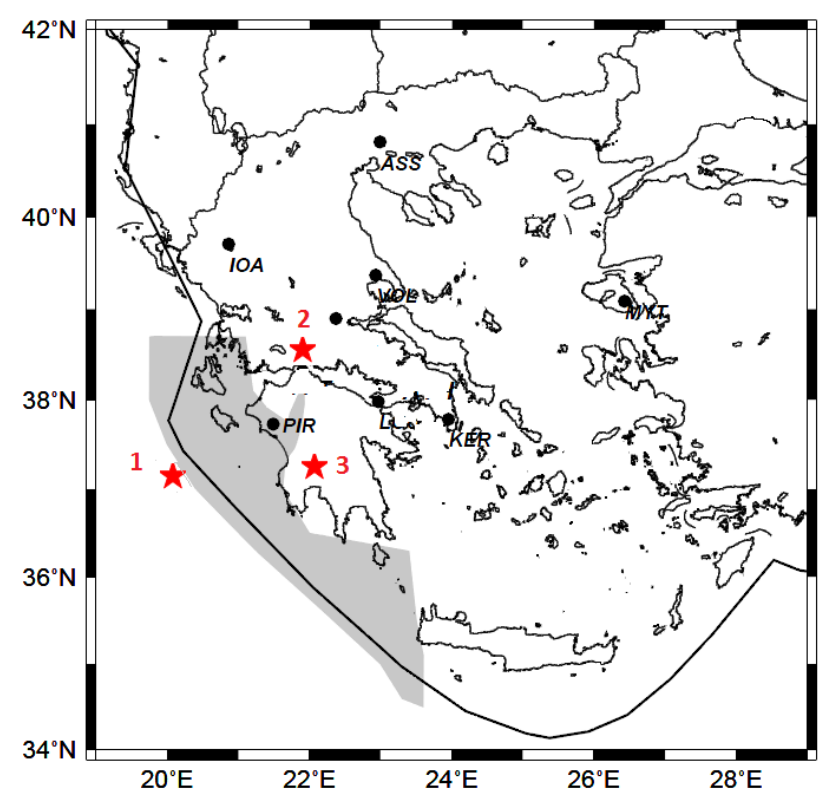

Fig. 2. The selectivity map (shaded area ) of PIR station. The epicenters of the three main shocks are denoted by red stars with numbers 1, 2 and 3 as listed in Table 1.

\section{Discussion}

The insertion of the new data sets of stress drop $\Delta \sigma_{B}$ and lead times $\Delta t$ in the previously derived power law relation

$\Delta \sigma_{B}=8.17 \Delta t^{-0.328,}$

which was based on 16 earthquakes and SES (Table 1 in Dologlou, 2010), results in the following exponents (Table 1): for the first set (no. 1 event) $\alpha_{1}=0.327$; for the second (no. 2 event) $\alpha_{2}=0.399$; and for the third one (no. 3 event) $\alpha_{3}=0.378$.

It is obvious that only the exponent $\alpha_{1}=0.327$, which corresponds to earthquake no. 1 and is almost the same with the reported one $\alpha=0.328$ by Dologlou (2010), matches the required value 0.33 (Surkov, 2002; Varotsos and Alexopoulos, 1984a) for critical behaviour. The other two events, no. 2 and no. 3, do not obey the relation expressed by Eq. (3) and lead to exponent values that differ markedly from the expected critical value. Thus, the relation of Eq. (3) is stable only when the appropriate set values of earthquake stress drop and SES lead time are introduced while it is significantly violated, when false earthquake-SES pairs are considered. That means that, except stability, this relation exhibits a high degree of sensitivity that may imply that it is not an artifact, but real physical dynamic processes evolving to criticality are present in the pre-focal area when the SES is emitted. An effort to explain the physics behind the interconnection between the lead time of SES and the stress drop of earthquakes has been attempted by the author Dologlou (2010) on the following basis: the lead time $\Delta t$ between the emission 


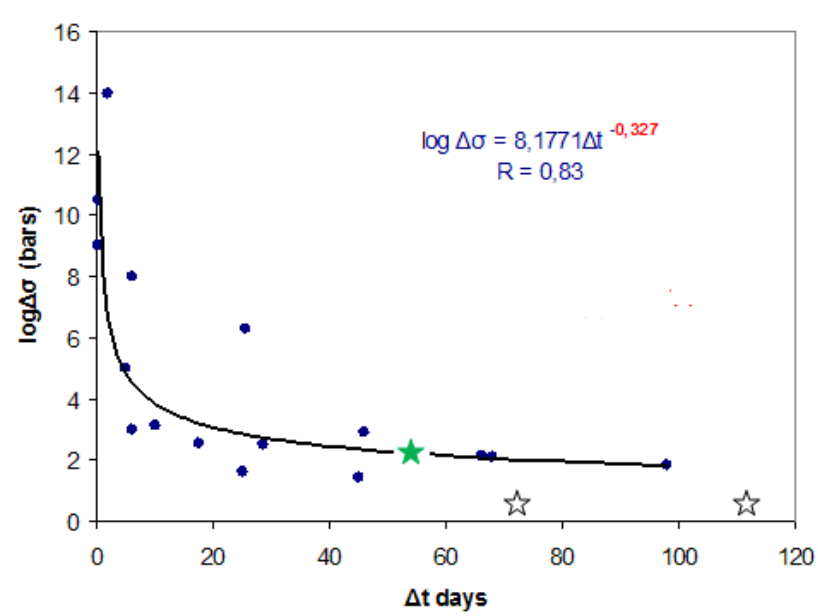

Fig. 3. The plot of the relation between the stress drop and the lead time for the earthquakes considered by Dologlou, 2010, along with the new data. A green star corresponds to the new event no. 1, while the open stars to the other two events no. 2 and 3 (Table 1). The derived power law relation along with its correlation coefficient $R$ are displayed on the top of the diagram.

of SES and the earthquake depends on the difference of the fracture stress $P_{\mathrm{fr}}$ for the earthquake occurrence and the critical stress $P_{\mathrm{cr}}$ for the SES emission, and on the stress rate $\beta$ as $\Delta t=\left(P_{\mathrm{fr}}-P_{\mathrm{cr}}\right) / \beta$. An increase of the stress rate, which may happen during the last preparatory stage of the earthquake process, leads to a decrease of the lead time since $P_{\mathrm{fr}}>P_{\mathrm{cr}}$. According to Varotsos et al. (1993a), and considering that in the same geotectonic area the fracture stress of rocks $P_{\mathrm{fr}}$ remains constant, an increase of the stress rate also causes an increase of the critical stress $P_{\text {cr }}$ and, thus, an enhanced decrease in the lead time. The question is whether the difference $\left(P_{\mathrm{fr}}-P_{\mathrm{cr}}\right)$ can be related to the stress drop of the earthquake, which is a topic for further investigation.

Another independent indication that event no. 1 must be the appropriate earthquake for the 25-26 May 2011 SES activity is the selectivity property of the SES signals, which states that a SES station can be sensitive to specific seismic regions while remaining inactive for others even at shorter distances (Varotsos and Lazaridou, 1991). The selectivity map of the PIR station (Sarlis et al., 2008), which detected the under study SES, is depicted in Fig. 2 (shaded area) along with the epicentres of the three earthquakes, which are denoted by red stars with numbers 1,2 and 3 as listed in Table 1. Only the epicentre of event no. 1 (earthquake on 19 July 2011) is located at the borders of the PIR selectivity map, while the other two fall clearly outside of it. Of course a selectivity map may be subjected to changes in time upon additional data.

\section{Conclusions}

New data from 2011, one SES activity and tree large earthquakes, were used to test the credibility of the power law relation of the form $\Delta \sigma_{B} \propto \Delta t^{-\alpha}$ (Dologlou, 2010) between the stress drop of earthquakes and the lead time of precursory SES. Here, we found that only the combination of data from the SES and the earthquake of 19 July 2011 obeyed the above power law with a critical exponent $\alpha=0.327$, a value that characterizes critical behavior and fully agrees with the reported one by Dologlou (2010). The combinations with the other two earthquakes failed to fulfil the above relation, resulting in exponent values far beyond criticality. Thus, this power law is very sensitive and the relevant critical exponent remains stable only for an appropriate set of data. That means this power law is not an artifact, but probably implies that real physical dynamic processes evolving to criticality are present in the pre-focal area when the SES is emitted. An attempt to complete the picture of the underlying physics in the interconnection of the stress drop and the lead time of the precursory SES is still in progress.

Edited by: M. E. Contadakis

Reviewed by: R. Teisseyre and another anonymous referee

\section{References}

Brune, J.: Tectonic stress and the spectra of seismic shear waves, J. Geophys. Res., 75, 4997-5009, 1970.

Brune, J. N.: Correction, J. Geophys. Res., 76, p. 5002, 1971.

Chen, K. C., Huang, W. G., and Wang, J. H.: Relationships Among Magnitudes and Seismic Moment of Earthquakes in the Taiwan Region, Terr. Atmos. Ocean. Sci., 18, 951-973, 2007.

Dologlou, E.: Power law relationship between parameters of earthquakes and precursory electrical phenomena revisited, Nat. Hazards Earth Syst. Sci., 9, 17-23, doi:10.5194/nhess-9-17-2009, 2009.

Dologlou, E.: Power law relationship between parameters of earthquakes and precursory electrical phenomena revisited II, Nat. Hazards Earth Syst. Sci., 10, 1403-1409, doi:10.5194/nhess-101403-2010, 2010.

Dologlou, E.: Comments on the generation mechanism of Seismic Electric Signals, Nat. Hazards Earth Syst. Sci., 11, 3093-3096, doi:10.5194/nhess-11-3093-2011, 2011.

Hanks, T. and Kanamori, H.: Moment magnitude scale, Geophys. Res., 84, 2348-2350, doi:10.1029/JB084iB05p02348, 1979.

Hanks, T. and Wyss, M.: The use of body wave spectra in the determination of seismic source parameters, Bull. Seism. Soc. Am., 62, 561-589, 1972.

Ishido, T. and Mizutani, H.: Experimental and theoretical basis of electrokinetic phenomena in rock-water systems and its applications to geophysics, J. Geophys. Res., 86, 1763-1775, 1981.

Keilis-Borok, V. I.: Fundamentals of earthquake prediction: four paradigms, in: Nonlinear Dynamics of the Lithosphere and Earthquake Prediction. Springer-Verlag, edited by: Keilis-Borok, V. I. and Soloviev, A. A., Berlin Heidelberg, 1-36, 2003. 
Kiratzi, A. A., Wagner, G. S., and Langston, C. A.: Source Parameters of Some Large Earthquakes in Nortern Aegean Determined by Body Waveform Inversion, Pure Appl. Geophys., 135, 515527, 1991.

Telesca, L., Lapenna, V., and Macchiato, M.: Multifractal fluctuations in seismic interspike series, Physica A, 354, 629-640, doi:10.1016/j.physa.2005.02.053, 2005.

Teotia, S. S. and Kumar, D.: Role of multifractal analysis in understanding the preparation zone for large size earthquake in the North-Western Himalaya region, Nonlin. Processes Geophys., 18, 111-118, doi:10.5194/npg-18-111-2011, 2011.

Sarlis, N., Skordas, E., and Varotsos, P.: Investigation of the seismicity after the initiation of a Seismic Electric Signal activity until the main shock, Proc. Jap. Acad. Ser. B, 84, 331-343, 2008.

Surkov, V., Uyeda, S., Tanaka, H., and Hayakawa, M.: Fractal Properties of medium and seismoelectric phenomena, J. Geodynamics, 33, 477-487, 2002.

Skordas, E., Sarlis, N., and Varotsos, P.: Effect of significant data loss on identifying electric signals that precede rupture by detrended fluctuation analysis in natural time, arXiv:cond. mat: 1003.1383v10, 26 January 2012.

Varotsos, P.: Comments on the formation entropy of a Frenkel defect in $\mathrm{BaF}_{2}$ and $\mathrm{CaF}_{2}$, Phys. Rev. B, 13, 938, doi:10.1103/PhysRevB.13.938, 1976.

Varotsos, P.: Comparison of models that interconnect point defect parameters, J. Appl. Phys., 101, 123503, doi:10.1063/1.2745359, 2007.

Varotsos, P. and Alexopoulos, K.: The curvature in conductivity plots of silver halides as a consequence of anharmonicity, J. Phys. Chem. Solids, 39, 759-761, 1978.

Varotsos, P. and Alexopoulos, K.: On the possibility of the enthalpy of a Schottky defect decreasing with increasing temperature, J. Physics C, 12, L761-L764, 1979.

Varotsos, P. and Alexopoulos, K.: Negative activation volumes of defects in solids, Phys. Rev. B, 21, 4898-4899, 1980.

Varotsos, P. and Alexopoulos, K.: Decisive importance of the bulk modulus and the anharmonicity in the calculation of migration and formation volumes, Phys. Rev. B, 24, 904-910, 1981.
Varotsos, P. and Alexopoulos, K.: Physical properties of the variations of the electric field of the earth preceding earthquakes, I, Tectonophysics, 110, 73-98, 1984a.

Varotsos, P. and Alexopoulos, K.: Physical properties of the variations of the electric field of the earth preceding earthquakes, II, Determination of epicentre and magnitude, Tectonophysics, 110, 99-125, 1984b.

Varotsos, P. and Alexopoulos, K.: Connection between the formation volume and formation Gibbs energy in noble gas solids, Phys. Rev. B, 30, 7305-7306, 1984c.

Varotsos, P. and Alexopoulos, K.: Thermodynamics of Point Defects and their Relation with the Bulk Properties, North Holland, Amsterdam, 1986.

Varotsos, P. and Lazaridou, M.: Latest aspects of earthquake prediction in Greece based on seismic electric signals, I, Tectonophysics, 188, 321-347, 1991.

Varotsos, P., Alexopoulos, K., Nomicos, K., and Lazaridou, M.: Earthquake prediction and electric signals, Nature, 322, 120 doi:10.1038/322120a0, 1986.

Varotsos, P., Alexopoulos, K., Nomicos, K., and Lazaridou, M.: Official earthquake prediction procedure in Greece, Tectonophysics, 152, 193-196, 1988.

Varotsos, P., Alexopoulos, K., and Lazaridou M.: Latest aspects of earthquake earthquake prediction in Greece based on Seismic Electric Signals II, Tectonophysics, 224, 1-37, 1993a.

Varotsos, P., Alexopoulos, K., Lazaridou M., and Nagao, T.: Earthquake predictions issued in Greece by Seismic Electric Signals since February 6, 1990, Tectonophysics, 224, 269-288, 1993b.

Varotsos, P. A., Sarlis, N. V., and Skordas, E. S.: Long-range correlations in the electric signals that precede rupture, Phys. Rev. E, 66, 011902, doi:10.1103/PhysRevE.66.011902, 2002.

Varotsos, P. A., Sarlis, N. V., and Skordas, E. S: Natural time analysis: the new view of time, Precursory Seismic Electric Signals, Earthquakes and other Complex Time-Series, Springer-Verlag, Berlin Heidelberg, 2011a.

Varotsos, P., Sarlis, N., and Skordas, E.: Scale-specific order parameter fluctuations of seismicity in natural time before main shocks, EPL, 96, 59002, doi:10.1209/0295-5075/96/59002, 2011 b. 